\title{
Ancestral chromosomes for the Peronosporaceae inferred from a telomere-to-telomere genome assembly of Peronospora effusa.
}

Kyle Fletcher ${ }^{1}$, Oon-Ha Shin ${ }^{2}$, Kelley J. Clark ${ }^{3,4}$, Chunda Feng ${ }^{4}$, Alexander I. Putmann ${ }^{5}$, James C. Correll, Steven J. Klosterman ${ }^{3}$, Allen Van Deynze ${ }^{2, *}$ and Richard Michelmore ${ }^{1,6, *}$

${ }^{1}$ The Genome Center, University of California, Davis, USA

${ }^{2}$ Seed Biotechnology Center, Department of Plant Sciences, University of California, Davis, USA

${ }^{3}$ USDA/U.S. Agricultural Research Station, 1636 East Alisal Street, Salinas, CA, USA

${ }^{4}$ Department of Entomology \& Plant Pathology, University of Arkansas, Fayetteville, AR, USA

${ }^{5}$ Department of Microbiology and Plant Pathology, University of California, Riverside, USA

${ }^{6}$ Departments of Plant Sciences, Molecular \& Cellular Biology, Medical Microbiology \& Immunology, University of California, Davis, USA

*Corresponding authors:

Richard Michelmore rwmichelmore@ucdavis.edu

Allen Van Deynze avandeynze@ucdavis.edu

\section{Keywords:}

oomycete, synteny, horizontal gene transfer, loss of heterozygosity, effector clusters, genome organization.

Funding: The work was supported by The Novozymes Inc. Endowed Chair in Genomics to RWM, and a grant received by AV from the California Seed Association Spinach Committee.

3

4

5

6

7

28

9




\section{Abstract}

31 We report the first telomere-to-telomere genome assembly for an oomycete. This assembly has extensive synteny with less complete genome assemblies of other oomycetes and will therefore serve as a reference genome for this taxon. Downy mildew disease of spinach, caused by the oomycete Peronospora effusa, causes major losses to spinach production. The 17 chromosomes of $P$. effusa were assembled telomere-to-telomere using Pacific Biosciences High Fidelity reads. Sixteen chromosomes are complete and gapless; Chromosome 15 contains one gap bridging the nucleolus organizer region.

37 Putative centromeres were identified on all chromosomes. This new assembly enables a re-evaluation of the genomic composition of Peronospora spp.; the assembly was almost double the size and contained more repeat sequences than previously reported for any Peronospora spp. Genome fragments consistently under-represented in six previously reported assemblies of $P$. effusa typically encoded repeats. Some genes annotated as encoding effectors were organized into multigene clusters on several chromosomes. At least two effector-encoding genes were annotated on every chromosome. The intergenic distances between annotated genes were consistent with the two-speed genome hypothesis, with some effectors located in gene-sparse regions. The near-gapless assembly revealed apparent horizontal gene transfer from Ascomycete fungi. Gene order was highly conserved between $P$. effusa and the genetically oriented assembly of the oomycete Bremia lactucae. High levels of synteny were also detected with Phytophthora sojae. Many oomycete species may have similar chromosome oomycetes. 


\section{Introduction}

The Peronosporaceae are a family in the Oomycota with hundreds of described species,

55 including Phytophthora and Peronospora spp. (Thines and Choi, 2016). Many species in the

56 Peronosporaceae are downy mildews, which are obligate biotrophs that are typically host-specific plant

57 pathogens. Other members of the Peronosporaceae are hemi-biotrophic, paraphyletic Phytophthora

58 species that typically have a wider host range than downy mildew species (Abad et al., 2019).

59 Phylogenetic analyses indicated that adaptation to obligate biotrophy from hemi-biotrophic ancestors

60 has occurred at least twice within the family, such that downy mildews are polyphyletic (Bourret et al.,

61 2018; Fletcher et al., 2018; Fletcher et al., 2019). Peronospora is the largest downy mildew genus and

62 contains over 400 species (Thines and Choi, 2016), including $P$. effusa, the subject of the current

63 analysis.

Downy mildew, caused by $P$. effusa, threatens spinach production because infection results in leaves unsuitable for sale and consumption. The disease is favoured by cool temperatures and high humidity and manifests as yellow lesions with blue-grey sporulation often on the abaxial surface of spinach leaves (Klosterman, 2016; Kandel et al., 2018). In conventional spinach production, the disease can be managed with use of fungicides and resistant cultivars; however, for organic production, deployment of resistant spinach cultivars is the only effective control available. Multiple resistance genes have been identified in the host, but the pathogen can rapidly adapt and evade host detection

71 (Correll et al., 2011; Kandel et al., 2018). To date, there are 19 named races of $P$. effusa; however,

72 isolates with variation in virulence phenotype are continuously identified and the biology behind the

73 emergence of new races is not clearly understood (Plantum, 2021, April 15). Previous genomic

74 investigations have yielded fragmented and repeat-sparse genome assemblies (Feng et al., 2018a;

75 Fletcher et al., 2018; Klein et al., 2020). The number of chromosomes the genome of $P$. effusa contained, the number and genomic distribution of genes encoding virulence factors, and how much of the genome 
77 is conserved or variable between isolates remained unknown. Genome assemblies are important

78 resources for determining the molecular basis for changes in virulence, resulting in the emergence of new pathotypes and provide informative loci for population studies.

In the current study, a 17-chromosome assembly is described for $P$. effusa that was generated using Pacific Biosciences High Fidelity (PacBio HiFi) reads. Sixteen telomere-to-telomere (T2T) gapless contigs and one T2T scaffold with a gap spanning the nucleolus organizer region were assembled. The genome was larger and more repeat-dense than anticipated and several new genes were annotated relative to previous assemblies. The content absent from previous assemblies was mostly long terminal repeat retrotransposons (LTR-RTs). There was a high degree of synteny between $P$. effusa, $P$. sojae, and

Bremia lactucae. Putative centromeres were identified through comparative genomics with $P$. sojae.

87 This is a landmark genome assembly for oomycete genomics.

\section{Results}

To determine the virulence phenotype of isolate UA202013, disease incidence was recorded on each of the standardized differential spinach lines. Disease incidence indicated that isolate UA202013 had a virulence pattern (Table 1), which did not match any of the 19 denominated races of $P$. effusa (Plantum, 2021, April 15).

The genome of isolate UA202013 of Peronospora effusa was assembled into 17 telomere-totelomere chromosomes. Sixteen of the chromosomes are complete and gapless; Chromosome (Chr.) 15 contains a single gap bridging the nucleolus organizer region (NOR). Chromosome assembly length ranged from 1.78 $\mathrm{Mb}$ (Chr. 9) to $8.42 \mathrm{Mb}$ (Chr. 1; Fig. 1). Putative centromeres were identified on all 17 chromosomes (Fig. 1); 15 putative centromeres contained at least one Copia-like transposon (CoLT) element (Supplementary Fig. 1). CoLT elements were previously hypothesized to be enriched in oomycete centromeres (Fang et al., 2020). Twelve P. effusa centromeres were syntenic with 
experimentally validated $P$. sojae centromeres (Fang et al., 2020), though some gene rearrangements were present around the centromeres of $P$. effusa Chromosomes 1, 6, 7, 8, and 15 (Supplementary Fig. 1). Putative centromeres of $P$. effusa Chr. 1 and Chr. 15 did not contain CoLTs but were syntenic with $P$. sojae centromeres (Supplementary Fig. 1). Telomeric satellite sequences (5'-CCCTAAA-3') were detected at both ends of every chromosome and ranged from $321 \mathrm{bp}$ to 1,283 bp. The GC content of the $P$. effusa assembly was $48.6 \%$, ranging from $49.2 \%$ to $48.1 \%$ per chromosome (Table 3 ). Within 100 kb windows, the AT content ranged from $60.9 \%$ to $43.1 \%$ (Fig. 1A). The assembly was scored by BUSCO (Simao et al., 2015 ) as $99.2 \%$ complete (232/234 models), of which $0.9 \%$ ( 2 models) were duplicated, using a protistspecific database.

The genome was $53.7 \%$ repetitive. The most common repeat elements were long terminalrepeat retrotransposons (LTR-RTs), comprising $40.2 \%$ of the genome. Chromosomes ranged from $43.1 \%$ to $67.1 \%$ repetitive sequence. Repeat content did not correlate with chromosome length (Table 2; Pearson's Correlation $=-0.38)$. The total genome size of $58.6 \mathrm{Mb}$ was approximately twice the size of previous assemblies (23.9 $\mathrm{Mb}$ to $32.1 \mathrm{Mb}$ ). Repeat content calculated for previous assemblies ranged from $14.4 \%$ to $39.0 \%$ (Table 3). Therefore, most of the size difference between the new assembly and previous assemblies is due to the repeat content assembled. Putative centromeres co-located with repetitive regions (Fig. 1). Pairwise alignment of 66,858 clustered repeat elements demonstrated that many repeats shared high identity ( $\geq 98 \%$; Supplementary Fig. 2 ). High identity alignments of repeats were found both within and between chromosomes (Supplementary Fig. 3). Nucleotide differences between LTR pairs of retrotransposons in the new assembly were less diverged than in previous $P$. effusa assemblies (Supplementary Fig. 4). Therefore, similar LTR-RTs assembled in short read assemblies were likely chimeric, pairing LTRs from different elements. This suggests that short-read assemblies are inadequate to date LTR expansion events accurately in oomycetes. Interestingly, the LTR divergence in 
123 the new HiFi assembly was comparable to that reported for $P$. ramorum and $P$. sojae, both assemblies

124 generated by Sanger sequencing (Tyler et al., 2006).

A total of 9,745 genes were annotated in the $P$. effusa genome, with an average of 166 genes

per $\mathrm{Mb}$. The number of genes annotated per chromosome ranged from 237 to 1,482 (Table 2) and

correlated with chromosome length $(P C=0.97)$. The density of genes ranged from 108 genes per $\mathrm{Mb}$ to

219 genes per $\mathrm{Mb}$. Gene density did not correlate with chromosome length $(P C=0.29)$. On average,

$27.6 \%$ of each $100 \mathrm{~kb}$ bin encoded genic sequence. Gene density was inversely related to repeat density

130

131

132

(Fig. 1). Of these genes, 307 were annotated as effectors. These included 98 crinkler effectors and 209

RXLR effectors. Effectors were encoded on every chromosome except Chr. 11 (Table 2). The effector

count per chromosome was weakly correlated with the chromosome length ( $P C=0.82)$. Some effectors were organized as clusters of genes with high identity (Fig. 1, Supplementary Fig. 5). Three clusters of crinkler annotations on Chr. 1, spanning approximately $141 \mathrm{~kb}, 34 \mathrm{~kb}$, and $84 \mathrm{~kb}$, were detected. There was a fourth, larger crinkler cluster on Chr. 10, spanning $523 \mathrm{~kb}$. Clusters of proteins annotated as having an RXLR-EER motif, WY domain, or both (RXLR-EER/WY) were identified on Chr. 2 (310 kb), Chr. 4 (1.1 Mb), Chr. 5 (745 kb), Chr. 14 (249 kb), Chr. 15 (245 kb), Chr. 17 (168 kb), and three independent clusters on Chr. 16 (182 kb, 360 kb, and 378 kb; Fig. 1; Supplementary Fig. 5). The RXLR-EER/WY clusters on Chr. 4 and Chr. 17 spanned centromeres that shared synteny with P. sojae (Supplementary Fig. 1). Inter-chromosome, lower identity alignments were detected for RXLR-EER/WY annotations, but not crinkler annotations (Supplementary Fig. 5). These inter-chromosomal alignments could be due to gene duplication and transposition. Crinkler clusters were composed of protein sequences sharing higher identity to one another than RXLR-EER/WY clusters (Supplementary Fig. 6). Inter-chromosomal alignments of effector proteins rarely shared high identity compared to intra-chromosomal alignments (Supplementary Fig. 6). 
Inferring orthology with the annotation of 37 other oomycetes from 34 different species

147 (Supplementary Table 1) assigned 8,739 annotations of P. effusa UA202013 to 6,425 orthogroups. An

148 additional 725 UA202013 proteins assigned to 483 orthogroups were identified as unique to $P$. effusa

149 and annotated in previous assemblies. Therefore, 281 P. effusa UA202013 annotations had no orthology

150 assigned with any previous oomycete assembly. Genes unique to $P$. effusa could be found on every

151 chromosome (Supplementary Fig. 7). Of the 1,006 genes unique to $P$. effusa, 174 were annotated as

152 effectors and a further 54 overlapped effector clusters.

The complete T2T genome assembly allowed for the investigation of genes unique to $P$. effusa

as potential horizontal gene transfer (HGT) events. An obvious signal for HGT was two neighbouring

gene models in the sub-telomeric region of Chr. 2 of $P$. effusa. The translated sequence of these two

gene models was highly homologous to several fungal proteins. The fungal homologs also neighboured

one another in their respective genome assemblies (Fig. 2A). One of these genes encoded a

metallophosphatase domain (MPD); the second gene did not encode a detectable conserved domain.

159 Both were annotated in previous P. effusa assemblies (Fletcher et al., 2018). The genes were embedded

160 in a repeat-rich region on Chr. 2, 22 kb and $\sim 64 \mathrm{~kb}$ away from flanking genes (Fig. 2A). Phylogenetically,

161 the $P$. effusa genes nested within fungal protein sequences, closest to the sunflower pathogen

162 Diaporthe helianthi and dieback associated fungi Valsa sordida and V. malicola (Fig. 2B, 2C). The flanking

163 genes on P. effusa Chr. 2 were assigned orthology with other oomycete proteins and branched as

164 expected phylogenetically (Fig. 2D, 2E). Flanking gene blocks were conserved between oomycete

165 assemblies, but rearrangements of the gene blocks were apparent (Fig. 2A). A paralogous MPD encoding

166 gene was present on $P$. effusa Chr. 15. There was no paralog for the second HGT candidate gene in this

167 region. The region on Chr. 15 was flanked by a block of oomycete genes and five copies of a repeated

168 sequence with homology to the flanking repeat on Chr. 2 (Supplementary Fig. 8). Additional MPD

169 encoding homologous genes were annotated on P. effusa Chr. 1 and Chr. 13. The intergenic distance 
flanking these genes was less than $500 \mathrm{bp}$. These genes were assigned orthology with other oomycete MPD encoding genes and branched as expected phylogenetically (Fig. 2). Therefore, MPD genes on Chr. 1 and Chr. 13 were not acquired by HGT. Genes encoding an MPD domain on Chr. 2 and Chr. 15 were likely acquired through a single HGT event from true fungi, followed by duplication. The read-depth of these genes in R15 and five other previously sequenced isolates was consistent with all four genes being present in all isolates sequenced (Supplementary Table 2).

Alignment of six previous $P$. effusa genome assemblies confirmed that most sequence missing from these previous assemblies was repeat encoding and not genic. The percentage of each $100 \mathrm{~kb}$ window covered by BLASTn alignments generated with other $P$. effusa assemblies was calculated, revealing poor representation in multiple windows across all chromosomes (Fig. 1E; Supplementary Fig. 9). This result is consistent with the previous assemblies being smaller than the new draft assembly (Table 3). Between 1,140 genes and 1,655 genes annotated in P. effusa UA202013 were absent in the assemblies of other isolates based on BLASTn alignments. As previously noted, the inflated gene count of $P$. effusa isolate Pfs1 is likely due to repeats annotated as genes (Klein et al., 2020). In total, 3,620 genes annotated in P. effusa UA202013 were not covered by BLASTn alignments from at least one other assembly of $P$. effusa. Only 121 genes were inferred as absent from all other assemblies of $P$. effusa (Supplementary Fig. 10). Further investigation revealed that 98 of the 121 proteins lacked orthology with any oomycete protein and may therefore be unique to isolate UA202013 of $P$. effusa or annotation artefacts.

Orthology analysis of 38 annotated oomycete genomes, including three from $P$. effusa, supported little novelty in the UA202013 assembly compared to P. effusa isolates R13 and R14. Isolate UA202013 was assigned to 97 multi-species orthogroups that did not contain R13 or R14 genes. Genes of all three isolates were assigned to a total of 6,264 orthogroups (Supplementary Fig. 11). These results suggest that most of the gene space is accessible through short-read assembly and that there are very 
194 few unique gene annotations specific to isolate UA202013. Further analysis of orthologs showed that

195 the increased gene count for UA202013 is due to collapsed paralogs in other assemblies. There were

196 several instances of orthogroups being assigned multiple annotations in the UA202013 assembly but

197 having fewer or no annotations in R13 or R14 assemblies (Supplementary Fig. 12). Analysis of read depth

198 supported the correct resolution of paralogs in the UA202013 assembly; high coverage of genes in

199 multiple orthogroups in R13 and R14 demonstrates that the paralogs were collapsed in the short-read

200 assemblies (Supplementary Fig. 13). Therefore, short-read assemblies are still a valuable resource for

201 obtaining the gene space of these organisms, though they are not sufficient to resolve the highly

202 repetitive architecture nor sufficient to resolve paralogous genes in the genome of $P$. effusa.

Read-depth analysis showed no copy number variation of whole chromosomes was present in

isolates UA202013, Pfs12, Pfs13, Pfs14, R13, and R14 (Fig. 1I; Supplementary Fig. 14). The only

back to the UA202013 assembly. This high coverage sequence encoded ribosomal subunits, consistent

207 with it being the NOR. This increased coverage across the NOR was detected in the reads of other

208 isolates (Fig. 1l; Supplementary Fig. 14). Additional significant peaks and troughs of coverage were

209 recorded for the five other isolates. Read depth differences correlated with the phenotypes, suggesting

210 the genomes of Pfs13 and R13 were similar, as were the genomes of Pfs12, Pfs14, and R14

211 (Supplementary Fig. 14). Interestingly, few reads for all five other isolates aligned to the effector cluster

212 on Chr. 14 of UA202013 (Supplementary Fig. 14, 15). This result suggests that the effectors encoded may

213 be absent or highly diverged in UA202013 versus the other five isolates. The four effectors annotated in

214 UA202013 contained a signal peptide prediction, an EER motif, two LWY domains, a partial GLY zipper

215 domain, and a partial electron transport complex protein domain (Supplementary Fig. 16). Between all

216 four, the N-terminus was more conserved than the C-terminus. The genomes of R13 and R14 each

217 contained a single homologous annotation, though in both cases the partial electron transport complex 
protein domain was not assembled as in the UA202013 gene models (Supplementary Fig. 16). No

significant depth variation was observed for other effector clusters in the UA202013 assembly

220 (Supplementary Fig. 14). Therefore, the cluster of putative effectors on Chr. 14 contains candidates for

221 proteins underlying the difference in virulence of isolate UA202013 compared to the other isolates. assembly of UA202013, seven genes were annotated as encoding effectors in the $750 \mathrm{~kb}$ region on Chr.

Therefore, one or more of these genes are good candidates for determining the avirulence phenotype. 
242

$3.1 \%$ of the genes are annotated as effectors; therefore, effectors were enriched in the gene-sparse regions of the assembly. The upper limit of this analysis was better than a previously reported similar analysis of $P$. effusa Pfs1 (Klein et al., 2020), likely because the Pfs1 assembly was more fragmented than the T2T UA202013 assembly. The two-speed genome hypothesis proposes that effectors are embedded in gene-sparse regions of the genome (Dong et al., 2015). Genes with $>50 \mathrm{~kb}$ intergenic regions were found on every chromosome, suggesting that every chromosome of $P$. effusa has potentially flexible regions involved in pathogenicity.

Comparative genomics demonstrated that the chromosomes of $P$. effusa were highly syntenic with assemblies of other oomycetes (Fig. 4A, 4B). The genome of B. lactucae has been ordered into 19 linkage groups (Fletcher et al., 2020), 15 of which were colinear with complete chromosomes of $P$. effusa (Fig. 4A). The other four B. lactucae linkage group (LG) scaffolds were syntenic with two chromosomes of P. effusa: LG 16 and LG 19 of B. lactucae were syntenic with Chr. 16 of $P$. effusa; LG 17 and LG 18 of B. lactucae were syntenic with Chr. 17 of $P$. effusa. Gene content was also conserved with P. sojae (Fig. 4B), though synteny of some scaffolds was found with multiple chromosomes (P. sojae 116 = P. effusa Chr. 1 and Chr. 13; P. sojae $115=$ P. effusa Chr. 6 and Chr. 10, P. sojae $117=$ P. effusa Chr. 9, Chr. 11, and Chr. 12). It remains unresolved whether these are true chromosomal fusions or assembly errors in $P$. sojae. Comparison to a more recent long-read assembly of $P$. sojae did not resolve this because the assembly was too fragmented (Supplementary Fig. 1). Phylogenetics of BUSCO genes found to be single copy across 31 oomycete assemblies supports polyphyly of downy mildews as reported previously (Bourret et al., 2018; Fletcher et al., 2018; Fletcher et al., 2019). The most recent common ancestor of $P$. effusa and $B$. lactucae is inferred as common to all downy mildews and Phytophthora species clades one to five (Fig. 4C). The most recent common ancestor of $P$. effusa and $P$. sojae (Phytophthora clade 7) is more ancient than the common ancestor of $P$. effusa and B. lactucae. Given 
the high levels of synteny between these three species, it is likely that the 17-chromosome architecture of $P$. effusa and B. lactucae will be ancestral to hundreds of other species in the Peronosporaceae.

\section{Discussion}

This report describes the first T2T assembly of an oomycete. Sixteen of the 17 chromosomes of

P. effusa are gapless; only Chr. 15 contains one gap, which likely encodes a highly repetitive rDNA array.

This assembly will be a key resource for comparative genomics with all other oomycetes and will advance the study of many important pathogens.

273 orientated genome assembly of $B$. lactucae (Fletcher et al., 2020). The $P$. effusa chromosomes were

274 numbered to match the corresponding chromosome-scale scaffolds of $B$. lactucae, reported previously

275 (Fletcher et al., 2020), because they have highly conserved single-copy gene contents, similar gene

276 orders along their chromosomes, and likely the same number of chromosomes (Fig. 4A). Therefore, the

277 common ancestor of $P$. effusa and B. lactucae may have had a similar chromosomal architecture.

278 Phylogenetic analysis indicated that downy mildews are polyphyletic (Fig. 4C); the most recent common

279 ancestor of biotrophic $P$. effusa and B. lactucae is also common to every downy mildew, as well as

280 Phytophthora clades 1 through 5 (Bourret et al., 2018; Fletcher et al., 2018; Fletcher et al., 2019). Given

281 the high levels of synteny with $P$. sojae, which is a clade 7 species, the proposed ancestral architecture

282 for many chromosomes is likely to be rooted deeper in the oomycete phylogeny than the last common

283 ancestor of downy mildews (Fig. 4C). Consequently, many downy mildew and Phytophthora species may

284 have the same ancestral chromosome configurations; therefore, this T2T assembly of $P$. effusa will serve

285 as a foundational reference for the study of Peronosporaceae and other oomycetes.

287 (Supplementary Fig. 1) and in the sub-telomeric region of Chr. 2 between $P$. effusa, $P$. viticola, and $P$. 
sojae (Fig. 2). The major structural variation observed between $P$. effusa and $P$. sojae (Fig. 4B) may be due to misassemblies in $P$. sojae or unique structural rearrangements in the evolutionary history of $P$. sojae. Interestingly, one misassembly in the $P$. sojae assembly that joins sequences syntenic to $P$. effusa

Chr. 6 and Chr. 10 has previously been reported (Fang et al., 2020). The generation of additional

chromosome-scale assemblies will be fundamental in determining how much structural variation exists within and between oomycete species and this T2T assembly of $P$. effusa provides the foundation for such investigations. double that of previous assemblies (Table 3). The increase in assembly size is attributed to the inability of short-reads and high-error rate of long reads to assemble high identity repeats and paralogs (Supplementary Fig. 12, 13). High identity repeats were collapsed, misassembled, or absent in previous short-read assemblies. Collapsed repeat sequences are reported to be present in genome assemblies of other oomycetes (Fletcher et al., 2019) and may result in unreliable analysis, including analysing bursts of LTR activity by nucleotide variation. Calculated LTR divergence since insertion on the assembly of $P$. effusa UA202013 compared to R13 and R14 (generated from short-reads) showed that the resulting 304 profiles differed between long- and short-read assemblies (Supplementary Fig. 4). This result is 305 consistent with short-read assemblies generating chimeric LTR-RTs with inflated divergence in their 306 LTRs. Therefore, estimations of LTR divergence since insertion in oomycete assemblies generated from 307 short-reads should be treated with care (Fletcher et al., 2019). 
of conserved domains characteristic of retrotransposons in the Pfs1 annotations confirmed the inclusion of LTR-RTs in gene annotations. Orthology analysis demonstrated that the increased gene count for

314 UA202013 was due to improved resolution of paralogs (Supplementary Fig. 12, 13) and not due to the

315 absence of single-copy genes from previous assemblies. These previously collapsed paralogs included

316 putative crinkler proteins, explaining the increase of this class of effector. Therefore, the generation of

317 short-read oomycete assemblies is still informative for comparative analyses because most single-copy

318 genes are recovered but paralogs may be underrepresented.

Short reads are also a valuable resource for comparative analysis between isolates to identify candidate regions containing molecular determinants of race phenotypes. Aligning reads of five other

321 isolates showed that read depth, nucleotide variation, and heterozygosity correlated genome-wide with 322 race phenotypes (Supplementary Fig. 14, 17, and 18). The two race 13 isolates sequenced independently across two studies are very similar, as are the two race 14 isolates. The SNP compositions of these isolates are consistent with somatic derivation from single founder genotypes. Race 14 was proposed to be derived from race 12 after a LOH event (Lyon et al., 2016). Our data support this and favour a LOH event in a region of Chr. 6, resulting in the loss of avirulence alleles (Supplementary Fig. 19). independent $\mathrm{LOH}$ events. Independent LOH events are consistent with Pfs12 (race 12 rather than race unique to UA202013. It is possible that the molecular determinant for UA202013 is located in this

332 cluster and is absent in races 12,13 , and 14 . Therefore, these effectors may be recognized by spinach 333 cultivars Clermont, Campania, or Lazio, which all carry the RPF4 locus. Functional studies are required to 334 test this hypothesis further. Other regions of the UA202013 assembly also had low read depth in the 335 other isolates but did not overlap clusters of effectors (Supplementary Fig. 14). 
The T2T assembly demonstrated that some high identity effectors were arranged as tight clusters in the genome (Fig. 1, Supplementary Fig. 5). However, RXLR-EER-WY proteins clustered on the same chromosome do not share $100 \%$ identity (Supplementary Fig. 6). Clusters of RXLR and CRN effectors have been described on unplaced scaffolds of Phytophthora spp. (Schornack et al., 2009)

340 Therefore, clusters of effectors are likely in other Peronosporaceae species, although they had not been 341 reported in the previous fragmented assemblies of Peronospora spp. Such clusters may have

342 consequences for the evolution of new virulence phenotypes because recombination within tightly

343 linked clusters, such as crinkler clusters, will be less frequent than for unlinked genes. The larger clusters 344 of RXLR-EER-WY effectors on the smaller chromosomes may have a frequency of recombination because 345 there will be a higher density of crossovers on the smaller chromosomes. More frequent recombination 346 within and between genes encoding effectors may result in the rapid evolution of novel virulence 347 phenotypes.

In contrast to clusters of genes encoding RXLR-EER-WY effectors, CRNs were tightly clustered on 349 larger chromosomes. CRNs within a cluster were more similar than clustered RXLR-EER-WY effectors 350 (Supplementary Fig. 6). Lower divergence implies that paralogous CRN encoding genes are diversifying 351 more slowly than RXLR-EER-WY encoding genes (Dong and Ma, 2021), reflecting different selection 352 pressures. Interestingly, transient expression of 15 P. sojae CRNs in Nicotiana benthamiana showed that 353 relatively few of the tested crinklers induced plant cell death (PCD); instead, most crinklers suppressed 354 PCD, consistent with CRNs acting as PCD regulators, not inducers (Shen et al., 2013; Amaro et al., 2017). by identifying an insertion of genes that are unique to $P$. effusa that lacked orthology with annotations 357 of other oomycete species. Several instances of HGT and subsequent duplication have been previously 358 described for the oomycetes, identifying HGT from fungi or bacteria to oomycetes (Belbahri et al., 2008; 359 Savory et al., 2015; McCarthy and Fitzpatrick, 2016). These used comparative analysis of assemblies for 
multiple oomycete species/isolates and focused on Phytophthora and Pythium species. Our comparison

of a complete genome to assemblies of other oomycetes provided robust evidence for the recent HGT

effusa. The functions of these genes are unknown; the duplicated gene encoded a metallophosphatase

371 had inter-genic distances less than $500 \mathrm{bp}$ and therefore are in the gene-dense regions of the genome.

372 The two speed-genome hypothesis postulates that genes in gene sparse-regions evolve faster (Dong et

373 al., 2015). Therefore, these horizontally acquired genes may be evolving differently from their homologs

374 with oomycete ancestry.

In summary, the T2T assembly of $P$. effusa provides an important foundation for comparative genomics between oomycete species and for analysis of diversity within $P$. effusa. Future studies will

377 determine how prevalent the 17-chromosome configuration is between diverse members of the

378 Peronosporaceae and whether there are major structural rearrangements in some lineages. It will also

379 be interesting to determine if the size and chromosomal locations of effector clusters vary, especially

380 between downy mildews, whose ancestors may have adjusted the number of effectors in their genome

381 independently while adaptation to biotrophy occurred. Finally, this assembly provides sequences which

382 can be tested through evolutionary, comparative, and functional analyses to determine what role they

383 play in the success of $P$. effusa. 


\section{Methods}

Isolate propagation and virulence phenotyping

Isolate UA202013 of $P$. effusa was collected from the spinach cultivar Sparrow in Thermal, CA on

from the growth chamber after 12 days to harvest sporangia. cultivars using a Badger Basic Spray Gun, Model 250. After inoculation, the plants were incubated in a

403 dew chamber for $24 \mathrm{~h}$, followed by a growth chamber for five days, and a final $24 \mathrm{~h}$ in the dew chamber

404 to induce sporulation. The cotyledons and true leaves of each plant were evaluated for downy mildew

405 disease symptoms seven days post-inoculation. Disease incidence (\%) for each cultivar was assigned

406 based on the number of plants showing chlorosis and sporulation out of the total number evaluated. 
Susceptible, resistant, and intermediate disease reaction evaluations were given based on disease

408 incidence.

409

\section{DNA extraction and library preparation}

Spore suspensions were centrifuged at $8,000 \mathrm{~g}$ for 5 minutes, the supernatant was removed,

412 and pellets frozen at $-80^{\circ} \mathrm{C}$. DNA was extracted from pellets (approx. $100 \mathrm{mg}$ ) using an OmniPrep kit (G-

413 Biosciences, St. Louis, MO) in $1 \mathrm{ml}$ lysis buffer (G-Biosciences) with $10 \mu \mathrm{l}$ proteinase $\mathrm{K}$ and digested for 1

414 hour at $60^{\circ} \mathrm{C}$ with periodic mixing to keep spores suspended in the lysis buffer. Library construction and

415 sequencing were done at the UC Davis Genome Center. DNA quantity and quality was verified with the

416 Quantus fluorometer assay (QuantiFluor ONE dsDNA Dye, Promega, Madison, WI, USA) and Femto

417 Pulse ${ }^{\circledR}$ Automated Pulsed Field (Agilent Technologies, Inc, Santa Clara, CA, USA), respectively. Sheared

418 gDNA (700 ng) was prepared with the Low Input DNA SMRTbell Express Template Prep Kit 2.0 (Pacific

419 Biosciences, Menlo Park, CA, USA) and loaded into a single 8M SMRT cell on a Pacific Biosciences Sequel

$420 \quad$ II.

421 Genome sequencing and assembly

PacBio HiFi reads were assembled with Hifiasm v0.14 (Cheng et al., 2021), generating 989

423 contigs, 11 of which were complete chromosomes. A $12^{\text {th }}$ chromosome (Chr. 10) was obtained from a

424 second Hifiasm assembly generating 57 contigs from reads filtered for mapping to oomycete assigned

425 contigs. Preliminary analysis was conducted by transferring annotations from P. effusa R13 (Fletcher et

426 al., 2018) onto the new assembly using Liftoff v1.3.0 (Shumate and Salzberg, 2020). Synteny of

427 intermediate assemblies with the genetically oriented assembly of B. lactucae (GCA_004359215.2)

428 (Fletcher et al., 2020) was determined using orthologs as calculated by OrthoFinder v2.2.1 (Emms and

429 Kelly, 2015). The initial 12 chromosomes were found to be colinear with $B$. lactucae linkage group- 
spanning scaffolds. Multiple $P$. effusa contigs were found to be colinear with other $B$. lactucae linkage

431 group-spanning scaffolds. Long reads aligned to these $P$. effusa contigs with minimap2 v2.17-r954-dirty

432 (Li, 2018) were assembled independently again with Hifiasm v0.14 (Cheng et al., 2021) and complete

were closed by aligning contigs generated by Hifiasm with contigs generated by HiCanu v2.0 (Nurk et al.,

435 2020), extending the Hifiasm contigs by $8,619 \mathrm{bp}$ and $12,694 \mathrm{bp}$, respectively, and adding telomeres to

the sequence. Two telomeric contigs of length 2,338,820 bp and 30,638 bp remained in the assembly,

437 both terminating in repetitive rDNA sequences. They could not be aligned, nor could reads be found that

bridged the sequences. Read alignment showed that the depth was high, indicating that a large repeat

Genome annotation was performed comparably to other $P$. effusa assemblies (Fletcher et al.,

442 2018). A repeat library was defined with RepeatModeler v1.73 (Smit and Hubley, 2008) and masked with

443 RepeatMasker v4.0.9 (Smit et al., 2013). Repeats were annotated in six other $P$. effusa genome

444 assemblies (Feng et al., 2018a; Fletcher et al., 2018; Klein et al., 2020) using the same repeat library.

445 Repeat sequences of UA202013 identified by RepeatMasker were clustered using CD-Hit v4.8.1 (Li and

446 Godzik, 2006), requiring 90\% identity to the centroid sequences. Pairwise identity within each cluster

447 was calculated using BLASTn v2.10.1 (Altschul et al., 1990). Inter- and intra-chromosomal clusters were

448 visualized using Circos v0.69-8 (Krzywinski et al., 2009). Nucleotide variation between LTRs assembled in

449 UA202013 was calculated as previously described (Fletcher et al., 2019). Briefly, LTR-RTs were identified

450 and annotated using LTRharvest v1.5.9 (Ellinghaus et al., 2008) and LTRdigest v1.5.9 (Steinbiss et al.,

451 2009), respectively. Internal domains were clustered with VMatch v2.3.0 and within cluster alignments

452 of 5' and 3' LTRs were generated with ClustalO v1.2.0 (Sievers et al., 2011). The divergence between LTR

453 pairs was calculated with BaseML v4.7b (Yang, 2007). Previous LTR divergence results for $P$. effusa, $B$. 
lactucae, $P$. infestans, P. ramorum, and P. sojae were taken from a previous analysis (Fletcher et al.,

2019). Results were plotted using R v4.0.1 base packages (R Development Core Team, 2012). calculated from the GFF file.

471 results were taken from the larger analysis containing annotations from 38 genome assemblies.

472 Orthogroups in which UA202013 contained five more genes than either R13 or R14 were considered

473 expanded. Read depth of the annotations in these orthogroups was calculated by aligning reads back to

474 their respective assemblies using bwa v0.7.17 (Li, 2013) or minimap2 v2.17-r954-dirty (Li, 2018) and

475 calculated with SAMtools v1.11 bedcov (Li et al., 2009). Stacked bar charts and dotplots were generated 
was calculate using BUSCO v2.0 with the protists_odb9 dataset (Simao et al., 2015). Translated single copy orthologs were added to the 18-BUSCO-gene concatenated alignment previously reported

479 (Fletcher et al., 2019). Protein alignments for each gene were re-calculated independent of one another using MAFFT v7.245 (Katoh and Standley, 2013). Alignments were concatenated and a single tree produced using RAxML v8.2.9 (Stamatakis, 2014), with 1,000 bootstraps and the PROTGAMMAAUTO model. The tree was visualized in Geneious v8.0.5 (Kearse et al., 2012). Horizontal gene transfer was investigated in the genes unique to $P$. effusa isolate UA202013 using BLASTp v2.10.1 (Altschul et al., 1990) to search the NCBI nr database excluding oomycetes. Genes with a low e-value were tested phylogenetically. A ClustalW alignment was built containing the candidate HGT gene and top BLASTp hits from non-oomycete and oomycete species. This alignment was used to build a neighbour-joining consensus tree to determine if the candidate gene was nested in nonoomycete sequences. A phylogenetic analysis was performed and visualized using Geneious v8.0.5 (Kearse et al., 2012). The same analysis was performed on flanking genes. Orthology of flanking genes 490 was investigated and plotted for contiguous oomycete genome assemblies using R v4.0.1 ( $R$ 491 Development Core Team, 2012), ggplot2 v3.3.3(Wickham, 2016), and ggforce v0.3.3 (Pedersen, 2019).

492 Chromosomal fragments of $P$. effusa Chr. 2 and Chr. 15 containing a duplicated gene were aligned 493 against each other using BLASTn. The alignment results were visualized using R v4.0.1 (R Development 494 Core Team, 2012) and ggplot2 v3.3.3 (Wickham, 2016). Synteny was plotted for the final $P$. effusa against $B$. lactucae and $P$. sojae by linking single copy orthologs between the assemblies with Circos v0.69-8 (Krzywinski et al., 2009). Twelve putative $P$. effusa 497 centromeres were inferred through synteny with the more fragmented $P$. sojae assembly GCA_009848525.1 for which coordinates of 12 centromeres have been previously defined (Fang et al., 499 2020). An additional five putative centromeres (Chr. 5, Chr. 7, Chr. 9, Chr. 12, and Chr. 13) were defined by identifying BLASTn hits (alignment length $>500$ bp) of a $P$. sojae CoLT previously hypothesized as 
501 space separated files and plotted using Circos v0.69-8 (Krzywinski et al., 2009).

diagnostic of oomycete centromeres (Fang et al., 2020). This alignment criterion was relaxed in called with FreeBayes v1.3.1-17-gaa2ace8 (Garrison and Marth, 2012). Bi-allelic single nucleotide Results were visualized using Heatmap2 from the R package gplots v3.1.1 (Warnes et al., 2020). The normalized read depth and coverage of previous draft genome assemblies per window were comparison to similar surveys searching for B. lactucae and $P$. citiricola centromeres (Fang et al., 2020).

Previous P. effusa genome assemblies (Feng et al., 2018a; Fletcher et al., 2020; Klein et al., 2020) were aligned to the new genome assembly using BLASTn (Altschul et al., 1990), filtering for alignments with a minimum length of 2,500 bp and scoring $\geq 95 \%$ identity. Genes for which $95 \%$ of the feature was within assembly-to-assembly alignments were reported as covered, regardless of the alignment length. Long reads of isolate UA202013 (this study) were aligned back to the assembly with Minimap2 v2.17r954-dirty (Li, 2018). Short reads of R13, R14 (Fletcher et al., 2018), pfs12, pfs13, and pfs14 (Feng et al., 2018a) were aligned with bwa v0.7.17 mem (Li, 2013). Variants for long and short read alignments were polymorphisms (SNPs) were used to cluster isolates genome-wide and per chromosome. Homozygous references were coded as 0 , heterozygous as 0.5 , and homozygous alternative as 1 . Euclidean distances between isolates were calculated using R v4.0.1 base function dist()(R Development Core Team, 2012).

For visualization, the genome was broken into $100 \mathrm{~kb}$ windows with a $25 \mathrm{~kb}$ step. The AT of each window was calculated using BEDTools2 v2.29.2 nuc (Quinlan, 2014). The density of repeats, genes, structural variants (UA202013 only), and SNPs was calculated using BEDTools2 v2.29.2 (Quinlan, 2014). calculated using BEDTools2 v2.29.2 (Quinlan, 2014). Per window heterozygosity was calculated as the number of SNPs genotyped 0/1 by FreeBayes v1.3.1-17-gaa2ace8. All tracks were formatted as white- 


\section{Acknowledgements}

525 We thank A. Garcia-Llanos (UC Davis) and A. Achieta (USDA/ARS, Salinas) for DNA extractions, O. Nguyen

526 (UC Davis) for library preparation and sequencing, H. Xu (UC Davis) for raw data submissions to NCBI,

527 and E. Georgian (UC Davis) for editorial services. The sequencing was carried out by the DNA

528 Technologies and Expression Analysis Cores at the UC Davis Genome Center, supported by NIH Shared

529 Instrumentation Grant 1S100D010786-01. The bioinformatic analysis was carried out using the UC Davis

530 LSSCO High Performance Computing cluster maintained by the UC Davis Bioinformatics Core.

\section{Author Contributions}

532 KF performed the bioinformatic analysis and drafted the manuscript. AP collected the isolate. OS, JC, CF,

$533 \mathrm{SK}$, and KC conducted the phenotyping of the isolate. AV conceived, coordinated, and provided funding

534 for sequencing. RM contributed to the bioinformatic analyses and to all drafts. All authors contributed

535 to the final manuscript and approved the submission.

\section{Competing Interests}

537 The authors declare no competing interests.

\section{Data availability}

539 The raw reads, genome assembly, and annotation are available under NCBI BioProject PRJNA745455. All

540 software used is described and cited in the Methods. 


\section{References}

543

544

545

546

547

548

549

550

551

552

553

554

555

556

557

558

559

560

561

562

563

564

565

566

567

568

569

570

571

572

573

574

575

576

577

578

579

580

581

582

583

584

585

586

587

Abad, Z., Burgess, T., Bienapfl, J., Redford, A., Coffey, M., and Knight, L. 2019. IDphy: Molecular and morphological identification of Phytophthora based on the types. USDA APHIS PPQ S\&T Beltsville Lab, USDA APHIS PPQ S\&T ITP, Centre for Phytophthora. Science and Management, and World Phytophthora Collection. https://idtools. org/id/phytophthora/index. php (accessed April 2021).

Altschul, S.F., Gish, W., Miller, W., Myers, E.W., and Lipman, D.J. 1990. Basic local alignment search tool. Journal of molecular biology 215:403-410.

Amaro, T.M.M.M., Thilliez, G.J.A., Motion, G.B., and Huitema, E. 2017. A Perspective on CRN Proteins in the Genomics Age: Evolution, Classification, Delivery and Function Revisited. Frontiers in plant science 8:99-99.

Belbahri, L., Calmin, G., Mauch, F., and Andersson, J.O. 2008. Evolution of the cutinase gene family: Evidence for lateral gene transfer of a candidate Phytophthora virulence factor. Gene 408:1-8.

Bourret, T.B., Choudhury, R.A., Mehl, H.K., Blomquist, C.L., McRoberts, N., and Rizzo, D.M. 2018. Multiple origins of downy mildews and mito-nuclear discordance within the paraphyletic genus Phytophthora. PLOS ONE 13:e0192502.

Campbell, M.S., Holt, C., Moore, B., and Yandell, M. 2014. Genome Annotation and Curation Using MAKER and MAKER-P. Current protocols in bioinformatics. 48:4.11.11-14.11.39.

Cheng, H., Concepcion, G.T., Feng, X., Zhang, H., and Li, H. 2021. Haplotype-resolved de novo assembly using phased assembly graphs with hifiasm. Nature Methods 18:170-175.

Correll, J., and du Toit, L. 2018. Guidelines for spinach downy mildew: Peronospora farinosa f. sp. spinaciae (Pfs) (= P. effusa). Collaboration for Plant Pathogen Strain Identification.

Correll, J.C., Bluhm, B.H., Feng, C., Lamour, K., du Toit, L.J., and Koike, S.T. 2011. Spinach: better management of downy mildew and white rust through genomics. European Journal of Plant Pathology 129:193-205.

Dong, S., and Ma, W. 2021. How to win a tug-of-war: the adaptive evolution of Phytophthora effectors. Current Opinion in Plant Biology 62:102027.

Dong, S., Raffaele, S., and Kamoun, S. 2015. The two-speed genomes of filamentous pathogens: waltz with plants. Current opinion in genetics \& development 35:57-65.

Ellinghaus, D., Kurtz, S., and Willhoeft, U. 2008. LTRharvest, an efficient and flexible software for de novo detection of LTR retrotransposons. BMC Bioinformatics 9:18.

Emms, D.M., and Kelly, S. 2015. OrthoFinder: solving fundamental biases in whole genome comparisons dramatically improves orthogroup inference accuracy. Genome Biology 16:157.

Fang, Y., Coelho, M.A., Shu, H., Schotanus, K., Thimmappa, B.C., Yadav, V., Chen, H., Malc, E.P., Wang, J., Mieczkowski, P.A., Kronmiller, B., Tyler, B.M., Sanyal, K., Dong, S., Nowrousian, M., and Heitman, J. 2020. Long transposon-rich centromeres in an oomycete reveal divergence of centromere features in Stramenopila-Alveolata-Rhizaria lineages. PLOS Genetics 16:e1008646.

Feng, C., Correll, J.C., Kammeijer, K.E., and Koike, S.T. 2013. Identification of New Races and Deviating Strains of the Spinach Downy Mildew Pathogen Peronospora farinosa f. sp. spinaciae. Plant Disease 98:145-152.

Feng, C., Lamour, K.H., Bluhm, B.H., Sharma, S., Shrestha, S., Dhillon, B.D.S., and Correll, J.C. 2018a. Genome Sequences of Three Races of Peronospora effusa: A Resource for Studying the Evolution of the Spinach Downy Mildew Pathogen. Mol Plant Microbe Interact 31:1230-1231.

Feng, C., Saito, K., Liu, B., Manley, A., Kammeijer, K., Mauzey, S.J., Koike, S., and Correll, J.C. 2018b. New Races and Novel Strains of the Spinach Downy Mildew Pathogen Peronospora effusa. Plant Disease 102:613-618. 
Fletcher, K., Zhang, L., Gil, J., Han, R., Cavanaugh, K., and Michelmore, R. 2020. AFLAP: Assembly-Free Linkage Analysis Pipeline using $k$-mers from whole genome sequencing data. bioRxiv:2020.2009.2014.296525.

Fletcher, K., Klosterman, S.J., Derevnina, L., Martin, F., Bertier, L.D., Koike, S., Reyes-Chin-Wo, S., Mou, B., and Michelmore, R. 2018. Comparative genomics of downy mildews reveals potential adaptations to biotrophy. BMC Genomics 19:851.

Fletcher, K., Gil, J., Bertier, L.D., Kenefick, A., Wood, K.J., Zhang, L., Reyes-Chin-Wo, S., Cavanaugh, K., Tsuchida, C., Wong, J., and Michelmore, R. 2019. Genomic signatures of heterokaryosis in the oomycete pathogen Bremia lactucae. Nature Communications 10:2645.

Garrison, E., and Marth, G. 2012. Haplotype-based variant detection from short-read sequencing. arXiv preprint arXiv:1207.3907.

Kandel, S.L., Mou, B., Shishkoff, N., Shi, A., Subbarao, K.V., and Klosterman, S.J. 2018. Spinach Downy Mildew: Advances in Our Understanding of the Disease Cycle and Prospects for Disease Management. Plant Disease 103:791-803.

Katoh, K., and Standley, D.M. 2013. MAFFT multiple sequence alignment software version 7: improvements in performance and usability. Mol Biol Evol 30.

Kearse, M., Moir, R., Wilson, A., Stones-Havas, S., Cheung, M., Sturrock, S., Buxton, S., Cooper, A., Markowitz, S., Duran, C., Thierer, T., Ashton, B., Meintjes, P., and Drummond, A. 2012. Geneious Basic: an integrated and extendable desktop software platform for the organization and analysis of sequence data. Bioinformatics 28:1647-1649.

Klein, J., Neilen, M., van Verk, M., Dutilh, B.E., and Van den Ackerveken, G. 2020. Genome reconstruction of the non-culturable spinach downy mildew Peronospora effusa by metagenome filtering. PLOS ONE 15:e0225808.

Klosterman, S. 2016. Spinach downy mildew-Threat, prevention and control. Prog. Crop Consult 1:1215.

Korf, I. 2004. Gene finding in novel genomes. BMC Bioinformatics 5:59.

Krzywinski, M.I., Schein, J.E., Birol, I., Connors, J., Gascoyne, R., Horsman, D., Jones, S.J., and Marra, M.A. 2009. Circos: An information aesthetic for comparative genomics. Genome Research 19:16391645.

$\mathrm{Li}, \mathrm{H}$. 2013. Aligning sequence reads, clone sequences and assembly contigs with BWA-MEM. arXiv preprint arXiv:1303.3997.

Li, H. 2018. Minimap2: pairwise alignment for nucleotide sequences. Bioinformatics 34:3094-3100.

Li, H., Handsaker, B., Wysoker, A., Fennell, T., Ruan, J., Homer, N., Marth, G., Abecasis, G., and Durbin, R. 2009. The Sequence alignment/map (SAM) format and SAMtools. Bioinformatics 25:2078-2079.

Li, W., and Godzik, A. 2006. Cd-hit: a fast program for clustering and comparing large sets of protein or nucleotide sequences. Bioinformatics 22.

Lyon, R., Correll, J., Feng, C., Bluhm, B., Shrestha, S., Shi, A., and Lamour, K. 2016. Population Structure of Peronospora effusa in the Southwestern United States. PLOS ONE 11:e0148385.

McCarthy, C.G.P., and Fitzpatrick, D.A. 2016. Systematic Search for Evidence of Interdomain Horizontal Gene Transfer from Prokaryotes to Oomycete Lineages. mSphere 1:e00195-00116.

Nurk, S., Walenz, B.P., Rhie, A., Vollger, M.R., Logsdon, G.A., Grothe, R., Miga, K.H., Eichler, E.E., Phillippy, A.M., and Koren, S. 2020. HiCanu: accurate assembly of segmental duplications, satellites, and allelic variants from high-fidelity long reads. Genome Research.

Pedersen, T.L. 2019. ggforce: Accelerating 'ggplot2'. R package version 0.22.

Plantum. (2021, April 15). Denomination of Pe: 18 and 19, two new races of downy mildew in spinach (Gouda, The Netherlands).

Quinlan, A.R. 2014. BEDTools: the Swiss-army tool for genome feature analysis. Current protocols in bioinformatics. 47:11.12.11-11.12.34. 
R Development Core Team. (2012). R: A language and environment for statistical computing (Vienna, Austria: R Foundation for Statistical Computing).

Savory, F., Leonard, G., and Richards, T.A. 2015. The role of horizontal gene transfer in the evolution of the oomycetes. PLoS pathogens 11:e1004805-e1004805.

Schornack, S., Huitema, E., Cano, L.M., Bozkurt, T.O., Oliva, R., Van Damme, M., Schwizer, S., Raffaele, S., Chaparro-Garcia, A., Farrer, R., Segretin, M.E., Bos, J., Haas, B.J., Zody, M.C., Nusbaum, C., Win, J., Thines, M., and Kamoun, S. 2009. Ten things to know about oomycete effectors. Molecular plant pathology 10:795-803.

Shen, D., Liu, T., Ye, W., Liu, L., Liu, P., Wu, Y., Wang, Y., and Dou, D. 2013. Gene Duplication and Fragment Recombination Drive Functional Diversification of a Superfamily of Cytoplasmic Effectors in Phytophthora sojae. PLOS ONE 8:e70036.

Shumate, A., and Salzberg, S.L. 2020. Liftoff: an accurate gene annotation mapping tool. Bioinformatics:btaa1016.

Sievers, F., Wilm, A., Dineen, D., Gibson, T.J., Karplus, K., Li, W., Lopez, R., McWilliam, H., Remmert, M., Söding, J., Thompson, J.D., and Higgins, D.G. 2011. Fast, scalable generation of high-quality protein multiple sequence alignments using Clustal Omega. Molecular Systems Biology 7:539539.

Simao, F.A., Waterhouse, R.M., loannidis, P., Kriventseva, E.V., and Zdobnov, E.M. 2015. BUSCO: assessing genome assembly and annotation completeness with single-copy orthologs. Bioinformatics 31:3210-3212.

Smit, A., and Hubley, R. 2008. RepeatModeler Open-1.0.

Smit, A., Hubley, R., and Green, P. 2013. RepeatMasker open-4.0.

Stamatakis, A. 2014. RAxML version 8: a tool for phylogenetic analysis and post-analysis of large phylogenies. Bioinformatics 30:1312-1313.

Steinbiss, S., Willhoeft, U., Gremme, G., and Kurtz, S. 2009. Fine-grained annotation and classification of de novo predicted LTR retrotransposons. Nucleic Acids Research 37:7002-7013.

Thines, M., and Choi, Y.J. 2016. Evolution, Diversity, and Taxonomy of the Peronosporaceae, with Focus on the Genus Peronospora. Phytopathology 106:6-18.

Tyler, B.M., Tripathy, S., Zhang, X., Dehal, P., Jiang, R.H., Aerts, A., Arredondo, F.D., Baxter, L., Bensasson, D., and Beynon, J.L. 2006. Phytophthora genome sequences uncover evolutionary origins and mechanisms of pathogenesis. Science (New York, N.Y.) 313.

Warnes, G., Bolker, B., Bonebakker, L., Gentleman, R., Huber, W., Liaw, A., Lumley, T., Maechler, M., Magnusson, A., Moeller, S., Schwartz, M., and Venables, B. 2020. gplots: Various R Programming Tools for Plotting Data.

Wickham, H. 2016. ggplot2: elegant graphics for data analysis. Springer.

Yang, Z. 2007. PAML 4: Phylogenetic Analysis by Maximum Likelihood. Molecular Biology and Evolution 24:1586-1591. 
675 Table 1. Disease incidence and reactions of spinach differential cultivars to isolate UA202013 of $P$.

676 effusa.

\begin{tabular}{rcc}
\hline $\begin{array}{c}\text { Differential } \\
\text { Line }\end{array}$ & $\begin{array}{c}\text { Disease } \\
\text { Incidence (\%) }\end{array}$ & $\begin{array}{c}\text { Disease } \\
\text { Reactions }^{b}\end{array}$ \\
\hline Viroflay & 100.0 & + \\
NIL5 & $26.7-70.0$ & $+/-$ \\
NIL3 & 100.0 & + \\
NIL4 & $15.4-64.3$ & $+/-$ \\
NIL6 & 100.0 & + \\
NIL1 & 0.0 & - \\
NIL2 & 0.0 & - \\
Pigeon & 0.0 & - \\
Caladonia & 0.0 & - \\
Meerkat & 0.0 & - \\
Hydrus & 0.0 & - \\
\hline
\end{tabular}

$677{ }^{a}$ Disease incidence is based on the number of plants (cotyledon or true leaves) showing chlorosis and/or 678 sporulation out of the total plants evaluated

$679{ }^{b}$ Differential lines were considered susceptible $(+)$ if disease incidence was greater than $85 \%$ or resistant 680 (-) if disease incidence was less than 15\%. The disease reaction was considered intermediate (+/-) if 681 disease incidence was between $15-85 \%$.

682 
Table 2. Summary statistics of chromosomes assembled for Peronospora effusa isolate UA202013.

\begin{tabular}{|c|c|c|c|c|c|c|c|c|c|}
\hline $\begin{array}{l}\text { P. effusa } \\
\text { UA202013 }\end{array}$ & $\begin{array}{l}\text { Assembled } \\
\text { length (bp) }\end{array}$ & $\begin{array}{l}\text { Total gaps } \\
\text { (bp) }\end{array}$ & $\begin{array}{l}\text { Percent } \\
\text { repeats }\end{array}$ & AT content & $\begin{array}{l}\text { Genes } \\
\text { annotated }\end{array}$ & Genes/Mb & $\begin{array}{l}\text { Effectors } \\
\text { annotated }\end{array}$ & $\begin{array}{l}\text { 5' Telomere } \\
\text { assembled } \\
\text { length (bp) }\end{array}$ & $\begin{array}{l}\text { 3' Telomere } \\
\text { assembled } \\
\text { length (bp) }\end{array}$ \\
\hline Chr. 1 & $8,417,896$ & 0 & $51.5 \%$ & $51.4 \%$ & 1482 & 176 & 70 & 706 & 685 \\
\hline Chr. 2 & $3,956,849$ & 0 & $53.5 \%$ & $51.7 \%$ & 596 & 151 & 23 & 597 & 958 \\
\hline Chr. 3 & $3,727,724$ & 0 & $49.5 \%$ & $50.9 \%$ & 666 & 179 & 10 & 723 & 616 \\
\hline Chr. 4 & $4,163,955$ & 0 & $51.4 \%$ & $51.3 \%$ & 751 & 180 & 28 & 803 & 695 \\
\hline Chr. 5 & $3,019,954$ & 0 & $57.3 \%$ & $51.5 \%$ & 393 & 130 & 16 & 680 & 1039 \\
\hline Chr. 6 & $4,409,438$ & 0 & $54.9 \%$ & $51.5 \%$ & 692 & 157 & 14 & 1283 & 649 \\
\hline Chr. 7 & $2,262,584$ & 0 & $67.1 \%$ & $51.5 \%$ & 261 & 115 & 6 & 756 & 703 \\
\hline Chr. 8 & $2,258,044$ & 0 & $56.3 \%$ & $51.6 \%$ & 334 & 148 & 5 & 502 & 656 \\
\hline Chr. 9 & $1,779,483$ & 0 & $49.7 \%$ & $51.8 \%$ & 315 & 177 & 3 & 682 & 523 \\
\hline Chr. 10 & $6,355,493$ & 0 & $48.6 \%$ & $51.3 \%$ & 1185 & 186 & 26 & 669 & 652 \\
\hline Chr. 11 & $2,566,028$ & 0 & $43.1 \%$ & $51.8 \%$ & 563 & 219 & 0 & 707 & 867 \\
\hline Chr. 12 & $2,778,632$ & 0 & $54.8 \%$ & $50.8 \%$ & 577 & 208 & 4 & 785 & 505 \\
\hline Chr. 13 & $2,196,953$ & 0 & $63.0 \%$ & $51.2 \%$ & 237 & 108 & 17 & 603 & 321 \\
\hline Chr. 14 & $2,153,540$ & 0 & $61.1 \%$ & $51.6 \%$ & 310 & 144 & 12 & 801 & 619 \\
\hline Chr. 15 & $2,369,557$ & 100 & $62.3 \%$ & $51.8 \%$ & 281 & 119 & 24 & 525 & 654 \\
\hline Chr. 16 & 2,944,237 & 0 & $54.1 \%$ & $51.3 \%$ & 479 & 163 & 27 & 795 & 784 \\
\hline Chr. 17 & $3,218,066$ & 0 & $52.8 \%$ & $51.9 \%$ & 623 & 194 & 22 & 708 & 553 \\
\hline Total & $58,578,433$ & 100 & $53.7 \%$ & $51.4 \%$ & 9745 & 166 & 307 & & \\
\hline
\end{tabular}


Table 3. Comparative assembly statistics of $P$. effusa isolate UA202013 with previously published assemblies of $P$. effusa.

\begin{tabular}{|c|c|c|c|c|c|c|c|c|c|c|}
\hline Isolate & Scaffold N50 & $\begin{array}{c}\text { Scaffold } \\
\text { count }\end{array}$ & Contig N50 & Contig count & $\begin{array}{l}\text { Assembly } \\
\text { size }\end{array}$ & Gaps & Repeats $^{1}$ & $\begin{array}{l}\text { Sequencing } \\
\text { technologies }\end{array}$ & $\begin{array}{c}\text { Reported } \\
\text { gene models }\end{array}$ & Study \\
\hline UA202013 & $3.7 \mathrm{Mb}$ & 17 & $3.7 \mathrm{Mb}$ & 18 & $58.6 \mathrm{Mb}$ & $<0.001 \%$ & $53.7 \%$ & PacBio HiFi & 9,745 & This study \\
\hline R13 & $72 \mathrm{~kb}$ & 784 & $48 \mathrm{~kb}$ & 1472 & $32.2 \mathrm{Mb}$ & $0.26 \%$ & $34.5 \%$ & Illumina & 8,607 & $\begin{array}{c}\text { (Fletcher et } \\
\text { al., 2018) }\end{array}$ \\
\hline R14 & $61 \mathrm{~kb}$ & 880 & $52 \mathrm{~kb}$ & 1275 & $30.8 \mathrm{Mb}$ & $0.56 \%$ & $32.0 \%$ & Illumina & 8,571 & $\begin{array}{c}\text { (Fletcher et } \\
\text { al., 2018) }\end{array}$ \\
\hline Pfs1 & $33.7 \mathrm{~kb}$ & 6762 & $33 \mathrm{~kb}$ & 6777 & $32.1 \mathrm{Mb}$ & $0.02 \%$ & $39.0 \%$ & $\begin{array}{c}\text { Illumina }+ \\
\text { PacBio }\end{array}$ & $13,277^{2}$ & $\begin{array}{c}\text { (Klein et al., } \\
2020)\end{array}$ \\
\hline Pfs12 & $21.3 \mathrm{~kb}$ & 4061 & $20.9 \mathrm{~kb}$ & 4505 & $25.2 \mathrm{Mb}$ & $0.2 \%$ & $17.5 \%$ & Illumina & $\begin{array}{c}\text { Not } \\
\text { Reported }\end{array}$ & $\begin{array}{c}\text { (Feng et al., } \\
\text { 2018a) }\end{array}$ \\
\hline Pfs13 & $18.1 \mathrm{~kb}$ & 4387 & $17.8 \mathrm{~kb}$ & 4939 & $24 \mathrm{Mb}$ & $0.26 \%$ & $14.4 \%$ & Illumina & $\begin{array}{c}\text { Not } \\
\text { Reported }\end{array}$ & $\begin{array}{c}\text { (Feng et al., } \\
\text { 2018a) }\end{array}$ \\
\hline Pfs14 & $22.1 \mathrm{~kb}$ & 4020 & $21.3 \mathrm{~kb}$ & 4488 & $24.9 \mathrm{Mb}$ & $0.22 \%$ & $16.6 \%$ & Illumina & $\begin{array}{c}\text { Not } \\
\text { Reported }\end{array}$ & $\begin{array}{c}\text { (Feng et al., } \\
\text { 2018a) }\end{array}$ \\
\hline
\end{tabular}

$687 \quad{ }^{1}$ Repeat content calculated in the present study using a repeat library calculated from P. effusa UA202013.

$688{ }^{2}$ Inflated gene model count due to some repeat elements annotated as genes.

689 


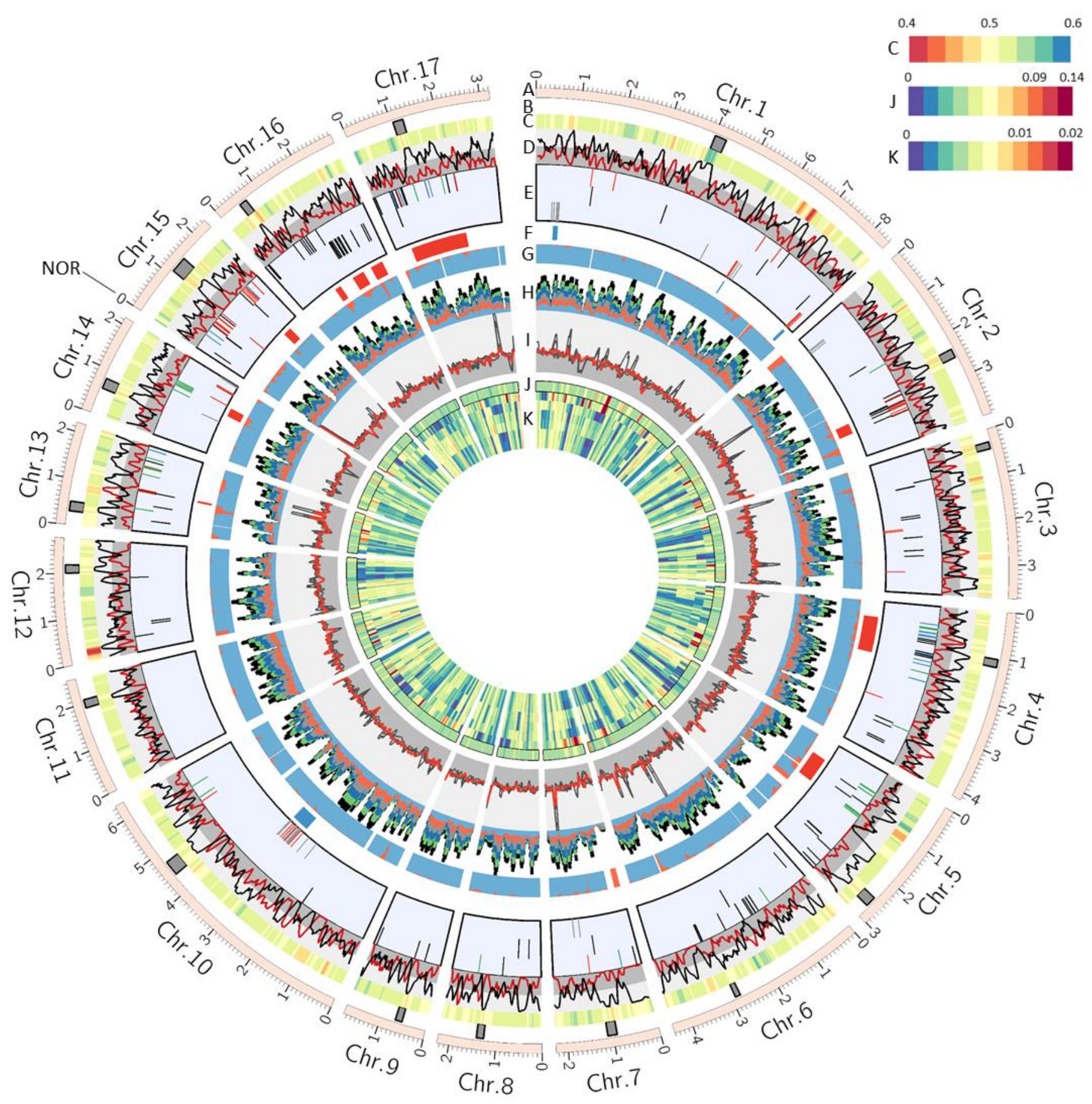

690 Fig. 1. Architecture of the 17 chromosomes of Peronospora effusa. A) Scaled chromosomes and ticks

691 showing lengths. Chromosome number was designated based on syntenic linkage groups with $B$.

692 lactucae. B) Dark grey boxes indicate putative positions of centromeres, some of which are syntenic with 
694 Heatmap of AT content, ranging from 0.4 to 0.6 . D) Gene density depicted in red, repeat density

695 depicted in black. The dark grey background shows 0 to 0.5 , light grey 0.5 to 1 . E) Effector annotations.

696 The first row are WY effectors; red ticks indicate the annotation contains a signal peptide prediction,

697 RXLR and EER motif and WY domain; blue ticks indicate annotation with a signal peptide and RXLR motif

698 and WY domain; green ticks indicate annotation with signal peptide and WY domain; black ticks indicate

699 a WY domain annotation with no signal peptide. In the second row are genes annotated as encoding

700 RXLR-EER proteins with a signal peptide (all black). Third row are crinkler annotations with (red) and

701 without (black) predicted signal peptides. F) Clusters of high identity crinkler (clue) and RXLR-EER/WY

702 (red) annotation. G) Orthology detected with 33 other oomycetes, blue indicates ortholog detected with

703 other species, red indicates genes unique to $P$. effusa, white indicates no annotation in a bin. $H$ )

704 Coverage of bin in six smaller P. effusa assemblies as determined by BLASTn. Colours indicate different

705 assemblies; all are Illumina-based except the bottom light blue, which was a hybrid Pac Bio and Illumina

706 assembly. Expanded in Supplementary Fig. 8. I) Normalized read depth of each bin, long reads of

707 UA202013 (33x) were plotted in red. Short reads of five other isolates were plotted in black. The

708 background indicated $0 x$ to $1 x$ (dark grey) and $1 x$ to $3 x$ (light grey). Expanded in Supplementary Fig. 14. J)

709 Density of structural variant calls inferred by Freebayes using the long reads of UA202013, plotted with

710 scale log base $=0.5$ K) Single nucleotide variant calls from alignments against UA202013 for alignments

711 of UA202013 long reads, and short reads of Pfs12, Pfs13, R13, Pfs14, and R14 plotted with scale log base

$712=0.5$. Heterozygosity is plotted in Supplementary Fig. 18. 
bioRxiv preprint doi: $h$ ttps://doi.org/10.1101/2021.09.14.460278; this version posted September 15,2021 . The copyright holder for this preprint (which was not certified by peer review) is the author/funder, who has granted bioRxiv a license to display the preprint in perpetuity. It is made available under aCC-BY-NC-ND 4.0 International license.
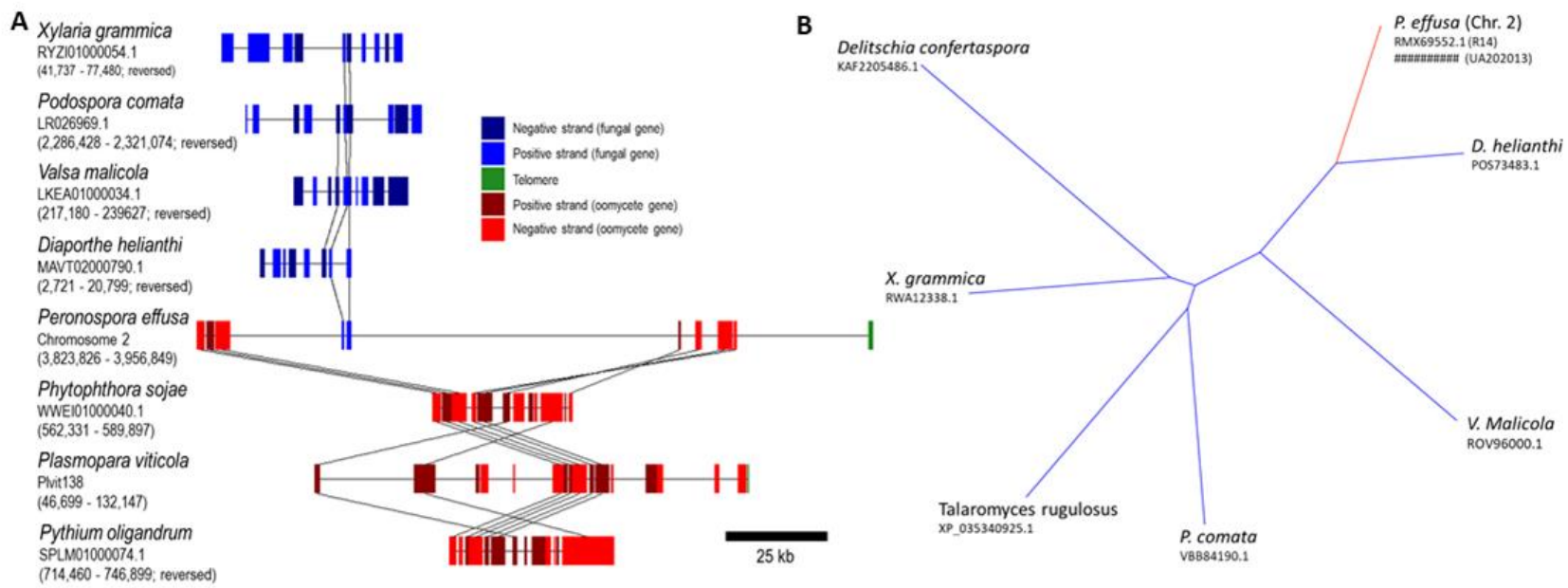

C
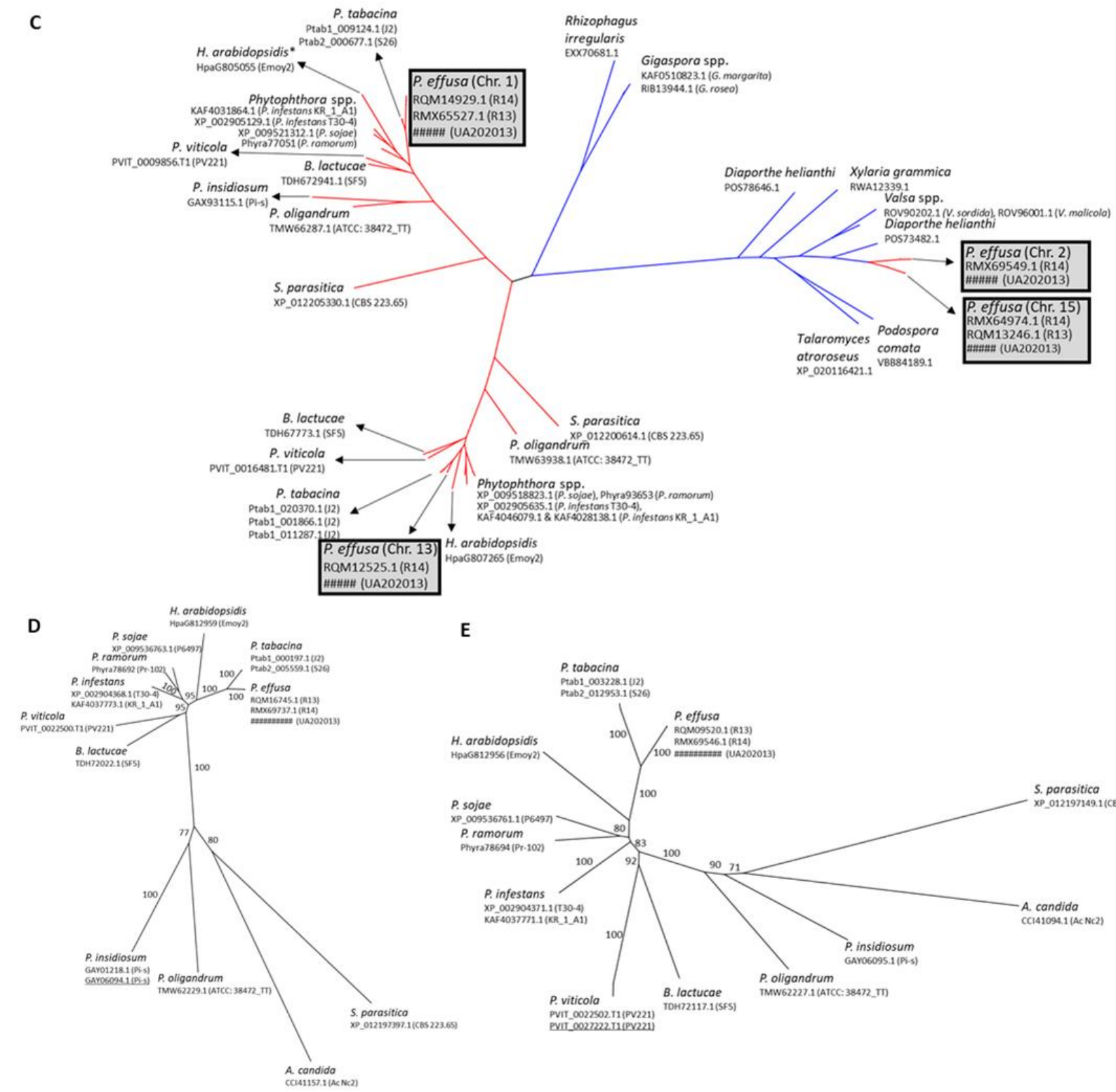

E

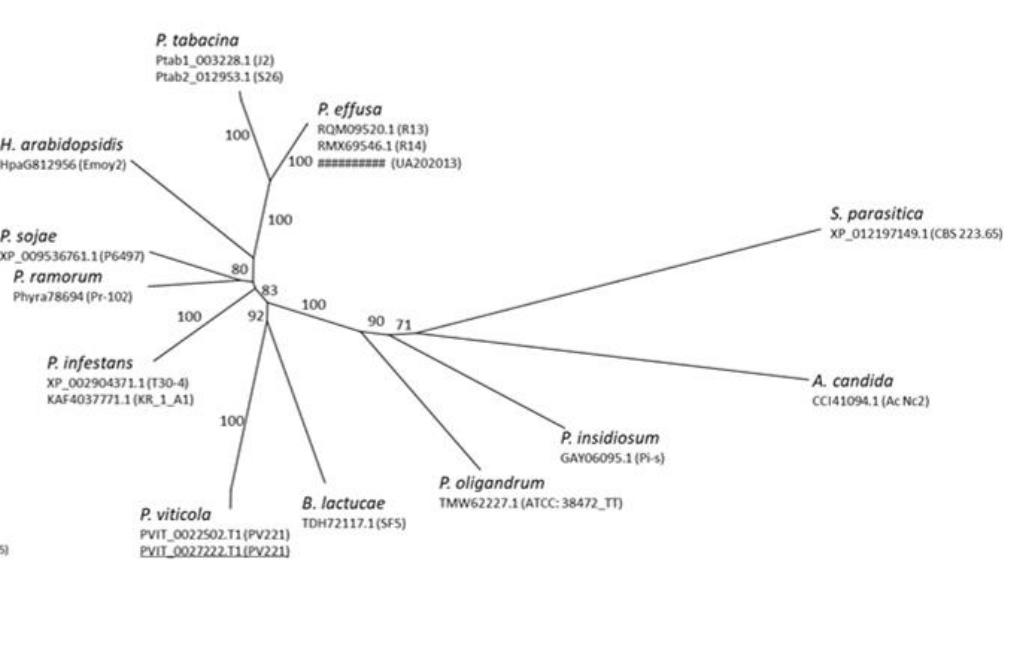


Fig. 2. Evidence of horizontal gene transfer in the genome of Peronospora effusa. A) Gene content of

716 several fungal and oomycete contigs or scaffolds. The top four sequences are true fungi and show

717 conserved gene order of two to three genes, linked by black lines. Two of those genes were found on

718 Chr. 2 of $P$. effusa, coloured blue and embedded in repeats. The red blocks indicate oomycete genes.

719 Orthologs of oomycete genes were found on single contigs/scaffolds in the genomes of other

720 oomycetes. The gene order of $P$. sojae and $P$. oligandrum was similar. Rearrangements were visible

721 when the $P$. sojae and $P$. oligandrum sequences were compared to $P$. effusa or $P$. viticola. B-E)

722 Neighbour joining trees of the horizontally acquired genes and the flanking gene. B) Only found in $P$.

723 effusa, the protein sequence was nested within fungal sequences. C) Several genes encoding proteins

724 containing metallophosphatase domains were found in the P. effusa genome. Homologs on Chr. 2 and

725 Chr. 15 were nested in fungal sequences; no orthologs were annotated in oomycete assemblies.

726 Orthologs in the genomes of other oomycetes were identified for the homologs on P. effusa Chr. 1 and

727 Chr. 13. Phylogenetic analysis of the glancing proteins ( $D$ and $E$ ) produced expected topologies.

728 Annotation of UA20213 submitted to NCBI and awaiting the assignment of accession identities. Similar

729 protein sequences can be found by using the NCBI accessions of P. effusa isolates R13 or R14. 

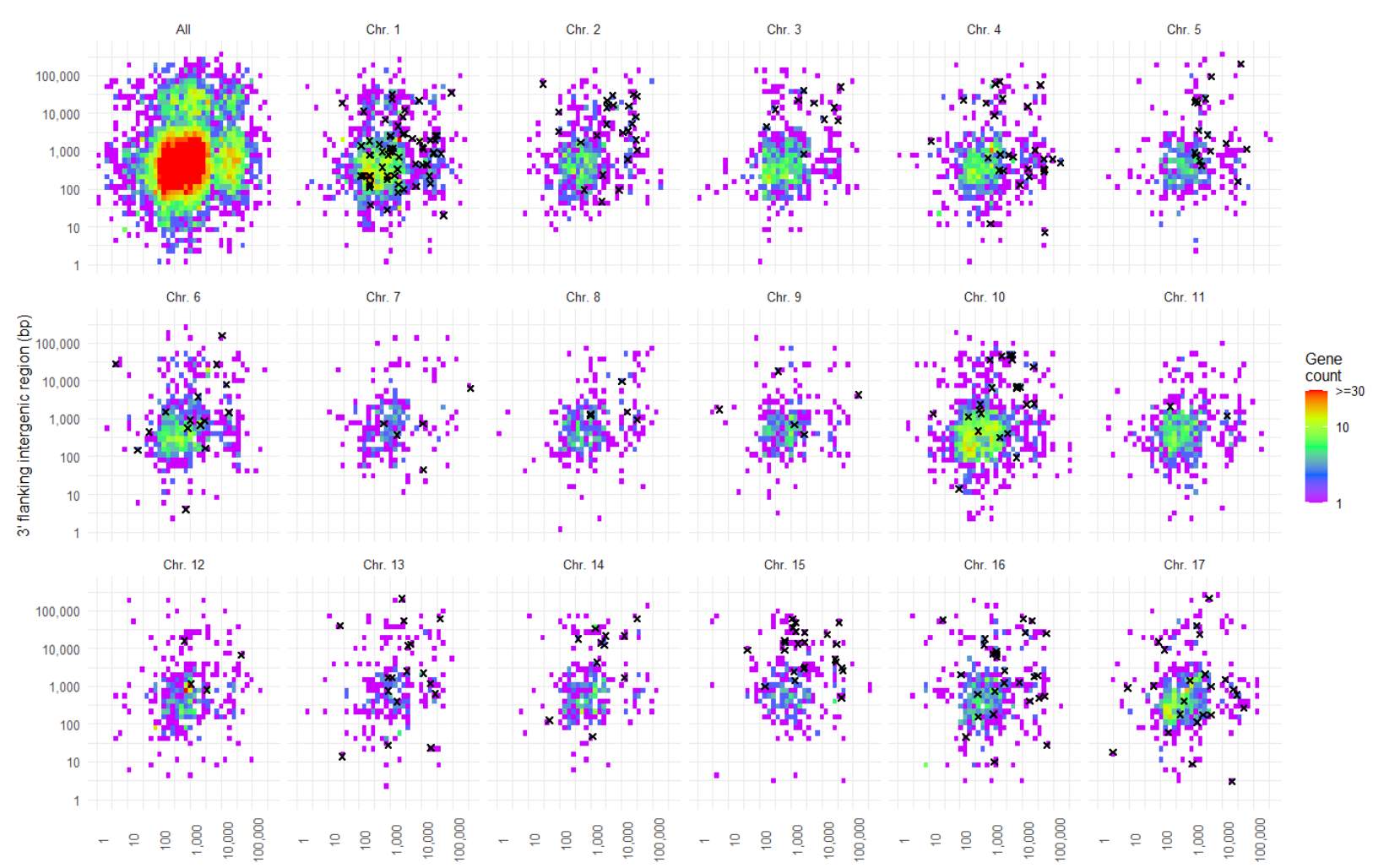

Fig. 3. Intergenic distances between annotated genes of Peronospora effusa isolate UA202013. For

each gene, the distance $5^{\prime}$ to the next gene are plotted on the $x$-axis and the distance $3^{\prime}$ to the next gene

$$
\text { are plotted on the } y \text {-axis. The top left plot shows the intergenic distance between all annotations }
$$

showing that the majority of genes cluster between $100 \mathrm{bp}$ and 5,000 bp on either side (coloured red). 
bioRxiv preprint doi: https://doi.org/10.1101/2021.09.14.460278; this version posted September 15, 2021. The copyright holder for this preprint (which was not certified by peer review) is the author/funder, who has granted bioRxiv a license to display the preprint in perpetuity. It is made available under aCC-BY-NC-ND 4.0 International license.
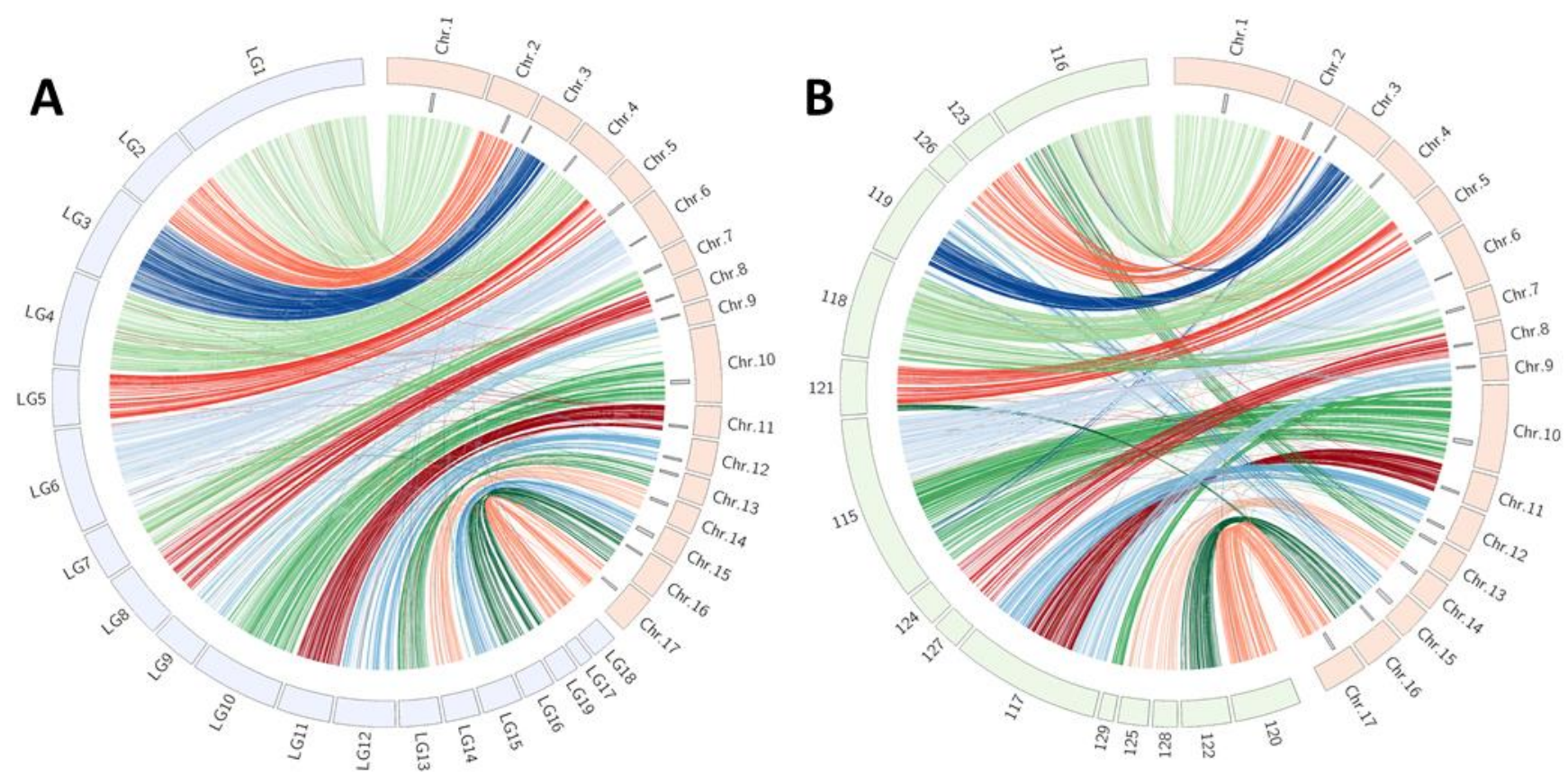

C

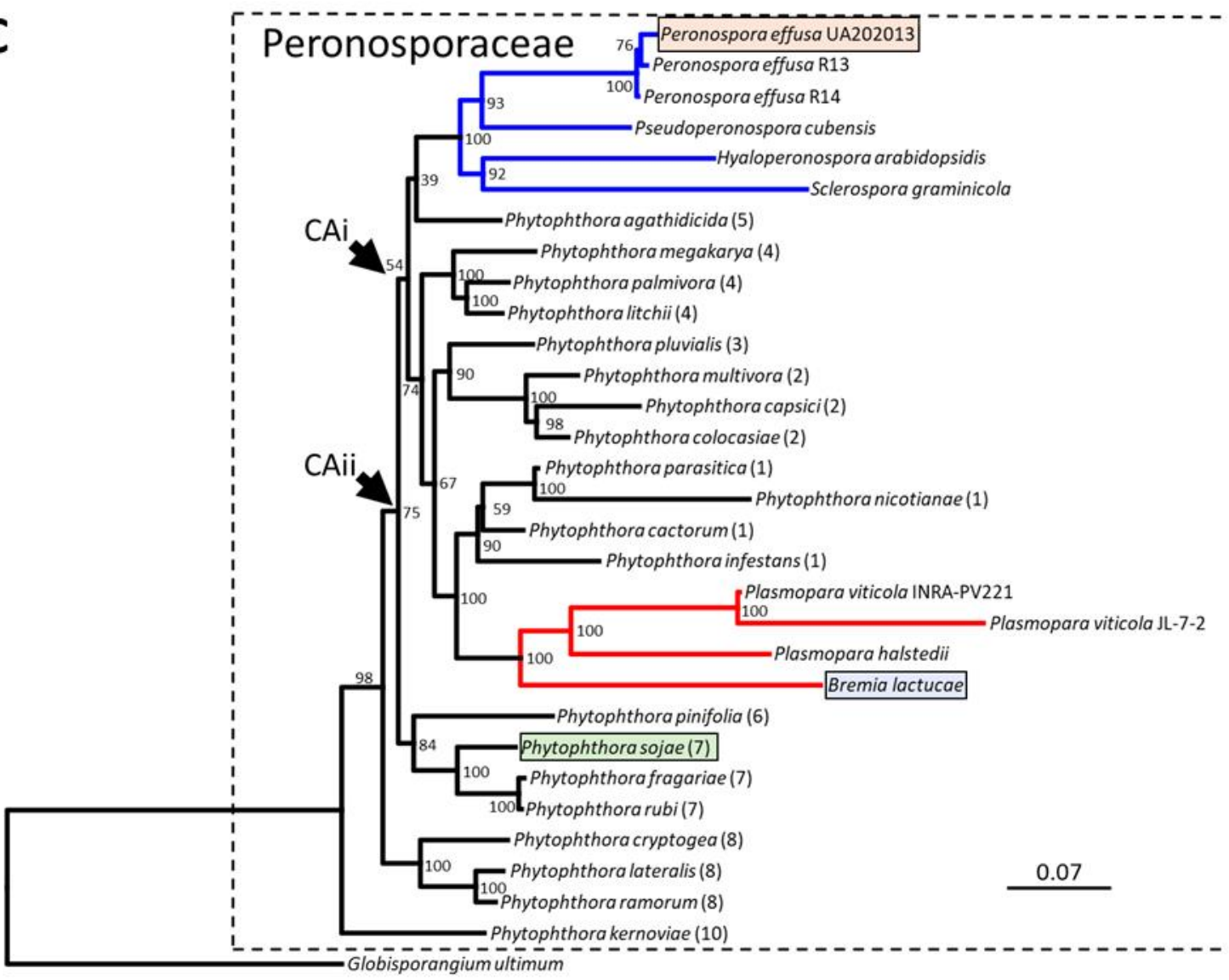

Fig. 4. The high synteny of Peronospora effusa chromosomes with other oomycetes. Synteny is 
741 A) nineteen genetically oriented scaffolds of the downy mildew $B$. lactucae (blue) and B) fifteen scaffolds

742 larger than $1 \mathrm{Mb}$ of the hemi-biotroph $P$. sojae (green). The plots are scaled to reflect the physical sizes

743 of the chromosomal sequences. C) Maximum likelihood phylogenetic tree calculated from the

744 concatenated alignment of 18 BUSCO proteins surveyed in 31 assemblies of 27 Peronosporaceae spp.

745 with Globisporangium ultimum (Pythiaceae) as an outgroup; multiple assemblies for $P$. effusa and $P$.

746 viticola were used. Branches coloured red and blue indicate the two polyphyletic downy mildew clades.

747 Species for which synteny was plotted are highlighted using the same colour palette as in A and B.

748 Parenthesized numbers indicate the Phytophthora clade a species has been assigned to. The most

749 recent common ancestor of $P$. effusa and $B$. lactucae is inferred as common to all downy mildews and

750 Phytophthora clades 1 to 5 (arrowed CAi). The most recent common ancestor of $P$. effusa and $P$. sojae is

751 inferred to be deeper in the phylogeny (arrowed CAii). Scale indicates the mean number of amino acid

752 substitutions per site. Branch support was calculated from 1,000 bootstraps. This figure was updated

753 from Fletcher et al. 2019, Figure 3. 